\title{
Yield of Screening for COVID-19 in Asymptomatic Patients Before Elective or Emergency Surgery Using Chest CT and RT-PCR (SCOUT) Multicenter Study
}

Citation for published version (APA):

Puylaert, C. A. J., Scheijmans, J. C. G., Borgstein, A. B. J., Andeweg, C. S., Bartels-Rutten, A., Beets, G. L., van Berge Henegouwen, M. I., Braak, S. J., Couvreur, R., Daams, F., van Es, H. W., Franken, L. C., Grotenhuis, B. A., Hendriks, E. R., de Hingh, I. H. J. T., Hoeijmakers, F., ten Holder, J. T., Huisman, P.' M., Kazemier, G., ... Stoker, J. (2020). Yield of Screening for COVID-19 in Asymptomatic Patients Before Elective or Emergency Surgery Using Chest CT and RT-PCR (SCOUT) Multicenter Study. Annals of Surgery, 272(6), 919-924. https://doi.org/10.1097/SLA.0000000000004218

Document status and date:

Published: 01/12/2020

DOI:

10.1097/SLA.0000000000004218

Document Version:

Publisher's PDF, also known as Version of record

Document license:

Taverne

Please check the document version of this publication:

- A submitted manuscript is the version of the article upon submission and before peer-review. There can be important differences between the submitted version and the official published version of record.

People interested in the research are advised to contact the author for the final version of the publication, or visit the DOI to the publisher's website.

- The final author version and the galley proof are versions of the publication after peer review.

- The final published version features the final layout of the paper including the volume, issue and page numbers.

Link to publication

\footnotetext{
General rights rights.

- You may freely distribute the URL identifying the publication in the public portal. please follow below link for the End User Agreement:

www.umlib.nl/taverne-license

Take down policy

If you believe that this document breaches copyright please contact us at:

repository@maastrichtuniversity.nl

providing details and we will investigate your claim.
}

Copyright and moral rights for the publications made accessible in the public portal are retained by the authors and/or other copyright owners and it is a condition of accessing publications that users recognise and abide by the legal requirements associated with these

- Users may download and print one copy of any publication from the public portal for the purpose of private study or research.

- You may not further distribute the material or use it for any profit-making activity or commercial gain

If the publication is distributed under the terms of Article 25fa of the Dutch Copyright Act, indicated by the "Taverne" license above, 


\title{
Yield of Screening for COVID-19 in Asymptomatic Patients Before Elective or Emergency Surgery Using Chest CT and RT-PCR (SCOUT)
}

\author{
Multicenter Study
}

Carl A. J. Puylaert, MD, PhD, ${ }^{*}$ Jochem C. G. Scheijmans, MD, $\dagger$ Alexander B. J. Borgstein, $M D, \ddagger$

Caroline S. Andeweg, MD, PhD, $\S$ Annemarieke Bartels-Rutten, MD, PhD, Geerard L. Beets, MD, PhD, $\|$

Mark I. van Berge Henegouwen, MD, PhD, $\ddagger$ Sicco J. Braak, MD, PhD, ${ }^{* *}$ Roy Couvreur, MD, $\dagger \dagger$

Freek Daams, $M D, P h D$, $\ddagger \ddagger$ Hendrik W. van Es, MD, PhD, $\S \S$ Lotte C. Franken, MD, $\uparrow$

Brechtje A. Grotenhuis, MD, PhD, || Eduard R. Hendriks, MD, ||| Ignace H. J. T. de Hingh, MD, PhD, ${ }^{* * *}$

Fieke Hoeijmakers, MD, $\dagger \dagger \dagger$ Joris T. ten Holder, MD, $\dagger \dagger \dagger$ Peter M. Huisman, MD, $\S \S \S$

Geert Kazemier, MD, PhD, $\dagger \dagger$ Floortje van Kesteren, $M D, P h D$, $\uparrow \uparrow$ Jurre van Kesteren, MD, $\dagger \dagger \dagger$

Kammy Keywani, BSc, $\ddagger$ Sara Z. Kuiper, MD, |||||| Maurits D. J. Lange, MD, || Mark E. Lobatto, MD, PhD, ${ }^{* * * *}$

Arthur W. F. du Mée, MD, $\dagger \dagger \dagger \dagger$ Martijn Poeze, MD, PhD, |||||| Elise M. van Praag, BSc, † Jorit van Rossen, MD, **

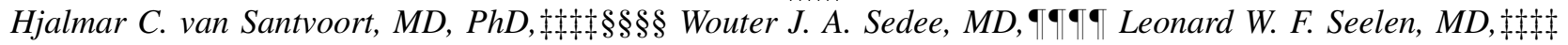

Sarah Sharabiany, MD, $\dagger$ Nico L. Sosef, MD, |||||||| Marian J. R. Quanjel, MD, PhD, ${ }^{* * * * *}$

Jeroen Veltman, MD, PhD, ${ }^{* *}$ Tim Verhagen, MD, $\dagger \dagger \dagger \dagger \dagger$ Vincent C. J. van de Vlasakker, MD, ${ }^{* * *}$

Pepijn D. Weeder, MD, |||||||| Jochem R. van Werven, MD, PhD, $\dagger \dagger \dagger \dagger$ Nina J. Wesdorp, MD, $\ddagger \dagger$

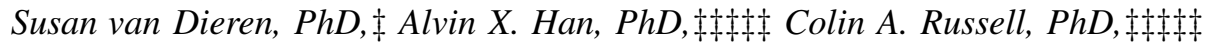

Menno D. de Jong, MD, PhD, §§§§§ Patrick M. M. Bossuyt, PhD, ฯฯฯ

Jet M. E. Quarles van Ufford, MD, PhD, |||||||||| Mathias W. Prokop, MD, PhD, ${ }^{* * * * * *}$

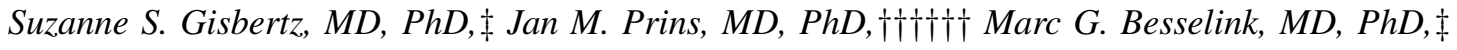

Marja A. Boermeester, MD, PhD, $\dagger$ Hester A. Gietema, MD, PhD, $\dagger \dagger \dagger \dagger \dagger \dagger$

Jaap Stoker, MD, PhD*凶, on behalf of the SCOUT study group

Objective: To determine the yield of preoperative screening for COVID-19 with chest CT and RT-PCR in patients without COVID-19 symptoms. Summary of Background Data: Many centers are currently screening surgical patients for COVID-19 using either chest CT, RT-PCR or both, due to the risk for worsened surgical outcomes and nosocomial spread. The optimal design and yield of such a strategy are currently unknown.

Methods: This multicenter study included consecutive adult patients without COVID-19 symptoms who underwent preoperative screening
From the *Department of Radiology and Nuclear Medicine, Amsterdam Gastroenterology Endocrinology Metabolism, Amsterdam UMC, University of Amsterdam, Amsterdam, the Netherlands; †Department of Surgery, Amsterdam Gastroenterology Endocrinology Metabolism, Amsterdam UMC, University of Amsterdam, Amsterdam, the Netherlands; †Department of Surgery, Cancer Center Amsterdam, Amsterdam UMC, University of Amsterdam, Amsterdam, the Netherlands; §Department of Surgery, St Jansdal Hospital, Harderwijk, the Netherlands; - Department of Radiology, Netherlands Cancer Institute, Amsterdam, the Netherlands; ||Department of Surgical Oncology, Netherlands Cancer Institute, Amsterdam, the Netherlands; ** Department of Radiology, Hospital Group Twente, Almelo, the Netherlands; ††Department of Surgery, Haaglanden Medical Center,

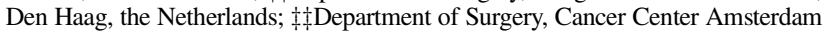
UMC, VU University Amsterdam, Amsterdam, the Netherlands; §§Department of Radiology, Sint Antonius Hospital, Nieuwegein, the Netherlands; $\uparrow$ Department of Surgery, Flevo Hospital, Almere, the Netherlands; ||||Department of Surgery, Tergooi Hospitals, Hilversum, the Netherlands; ${ }^{* * *}$ Department of Surgery, Catharina Hospital, Eindhoven, the Netherlands; $+\dagger \dagger$ Department of Surgery, Dijklander

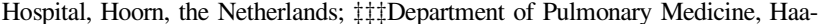

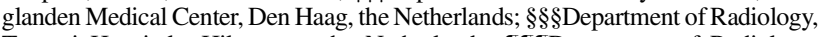
Tergooi Hospitals, Hilversum, the Netherlands; $\uparrow \uparrow$ Department of Radiology, Amsterdam UMC, University of Amsterdam, Amsterdam, the Netherlands; ||||||Department of Surgery, Maastricht UMC+, Maastricht, the Netherlands; ****Department of Radiology, Spaarne Gasthuis, Haarlem and Hoofddorp, the Netherlands; †††Department of Radiology, Amphia Hospital, Breda, the

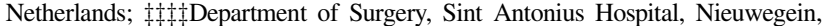
the Netherlands; $\S \S \S \S$ Department of Surgery, UMC Utrecht Cancer Center, UMC Utrecht, Utrecht, the Netherlands; $\uparrow \uparrow$ Department of Emergency Medicine, St Jansdal Hospital, Harderwijk, the Netherlands; ||||||||Department of Surgery, Spaarne Gasthuis, Haarlem and Hoofddorp, the Netherlands; ${ }^{* * * * *}$ Department of Pulmonary Medicine, Sint Antonius Hospital, Nieuwegein, the Netherlands; $\dagger \dagger \dagger \dagger \dagger$ Department of Surgery, Hospital Group Twente, Almelo, the Netherlands.; 括位aboratory of Applied Evolutionary Biology, Amsterdam UMC, University of Amsterdam, Amsterdam, the Netherlands; $\$ \S \S \S \S$ Department of Medical Microbiology and Infection Prevention, Amsterdam UMC, University of Amsterdam, Amsterdam, the Netherlands; $9 \uparrow$ Department of Clinical Epidemiology, Biostatistics and Bioinformatics, Amsterdam UMC, University of Amsterdam, Amsterdam, the Netherlands; ||||||||||Department of Radiology, Haaglanden Medical Center, Den Haag, the Netherlands; ${ }^{* * * * * * D e p a r t m e n t ~ o f ~ R a d i o l o g y, ~ R a d b o u d ~}$ UMC, Nijmegen, the Netherlands; $\dagger+\dagger \dagger \dagger \dagger$ Department of Internal Medicine, Division of Infectious Diseases, Amsterdam Institute for Infection and Immunity (AI\&II), Amsterdam UMC, University of Amsterdam, Amsterdam, the

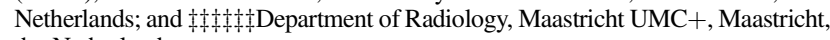
the Netherlands.

凶j.stoker@amsterdamumc.nl.

C.A.J.P., J.C.G.S., and A.B.J.B. shared first authorship and contributed equally to this article.

S.S.G., J.M.P., M.G.B., M.A.B., H.A.G., and J.S. shared last authorship and contributed equally to this article.

De Hingh has an unrestricted research fund from RanD Biotech and Roche for research non-related to current manuscript and paid to the institute. Van Berge Henegouwen is consultant for Mylan, Johnson and Johnson and received research grants from Olympus and Stryker paid to the institute.

No external funding was received for this study.

The authors report no conflicts of interest.

A complete list of the investigators in the SCOUT study group is given in the Supplementary Material, http://links.lww.com/SLA/C315.

Supplemental digital content is available for this article. Direct URL citations appear in the printed text and are provided in the HTML and PDF versions of this article on the journal's Web site (www.annalsofsurgery.com).

Copyright (C) 2020 Wolters Kluwer Health, Inc. All rights reserved.

ISSN: 0003-4932/20/27206-0919

DOI: $10.1097 /$ SLA.0000000000004218 
using chest CT and RT-PCR before elective or emergency surgery under general anesthesia.

Results: A total of 2093 patients without COVID-19 symptoms were included in 14 participating centers; 1224 were screened by CT and RT-PCR and 869 by chest CT only. The positive yield of screening using a combination of chest CT and RT-PCR was $1.5 \%$ [95\% confidence interval (CI): $0.8-2.1]$. Individual yields were $0.7 \%$ (95\% CI: $0.2-1.1$ ) for chest CT and 1.1\% (95\% CI: 0.6-1.7) for RT-PCR; the incremental yield of chest CT was $0.4 \%$. In relation to COVID-19 community prevalence, up to $\sim 6 \%$ positive RT-PCR was found for a daily hospital admission rate $>1.5$ per 100,000 inhabitants, and around $1.0 \%$ for lower prevalence.

Conclusions: One in every 100 patients without COVID-19 symptoms tested positive for SARS-CoV-2 with RT-PCR; this yield increased in conjunction with community prevalence. The added value of chest CT was limited. Preoperative screening allowed us to take adequate precautions for SARS$\mathrm{CoV}-2$ positive patients in a surgical population, whereas negative patients needed only routine procedures.

Keywords: computed tomography, COVID-19, preoperative screening, RTPCR

(Ann Surg 2020;272:919-924)

A fter the peak of the COVID-19 outbreak, hospitals around the world are now increasing their elective surgical care. ${ }^{1,2}$ The question whether to screen asymptomatic patients before surgery for COVID-19 remains unanswered.

Patients infected with SARS-CoV-2 have increased risk of postoperative complications and mortality. ${ }^{3-5}$ In addition, surgical patients with undetected COVID-19 could potentially shed SARS$\mathrm{CoV}-2$, placing hospital workers at risk, particularly during intubation and other aerosolizing procedures. ${ }^{6-9}$ Furthermore, if not isolated, patients may infect other hospitalized patients, of whom many are prone to developing severe COVID-19 due to older age and comorbidities. ${ }^{10,11}$ In a recent international survey, up to $59 \%$ of 264 centers from 37 countries worldwide reported to screen patients scheduled for pancreatic surgery, using chest computed tomography (CT) and/or reverse-transcriptase polymerase chain reaction (RTPCR). ${ }^{12}$ Given the limited resources, additional costs of screening, the burden of ionizing radiation, and the increase of non-COVID surgery there is an urgent need to evaluate the effectiveness of preoperative screening for COVID-19. Secondly, the effectiveness of preoperative screening in relation to changes in COVID-19 community prevalence should be explored. The Infectious Diseases Society of America guideline on COVID-19 diagnosis recently advised preoperative screening using SARS-CoV-2 RT-PCR in all asymptomatic individuals undergoing surgery. ${ }^{13}$

In the Netherlands, the first COVID-19 patient was identified on February 27, 2020, followed by a rapid increase in the number of confirmed patients. ${ }^{11}$ In the following weeks, many hospitals started routine preoperative screening in asymptomatic surgical patients as a method to detect asymptomatic COVID-19. Early routine screening was performed with chest CT only. Facilitated by improved availability of RT-PCR in later weeks, a nationwide Dutch guidance protocol was released that advised preoperative screening with chest CT and RT-PCR.

The effectiveness of this preoperative screening protocol in asymptomatic patients is unclear as RT-PCR testing is usually only performed in symptomatic patients. RT-PCR may be prone to sampling error and asymptomatic patients may have lower viral load than symptomatic COVID-19 patients. ${ }^{14-17}$ Moreover, chest CT is not recommended for screening in asymptomatic patients, ${ }^{18}$ although up to $63 \%$ of asymptomatic COVID-19 patients are reported to have abnormalities on chest $\mathrm{CT}^{8,19,20}$
The SCOUT study aimed to evaluate the yield of preoperative screening for COVID-19 using chest CT and RT-PCR in adult patients without COVID-19 symptoms, scheduled for elective or emergency surgical or other interventional procedures under general anesthesia.

\section{METHODS}

\section{Study Oversight}

The multicenter observational SCOUT study was conducted at 3 academic and 11 nonacademic hospitals in the Netherlands. Because of the observational nature of the study, formal approval was waived by the institutional review board of the Amsterdam $\mathrm{UMC}$, location AMC. This was endorsed by the institutional review board at each participating center. Informed consent was obtained through an opt-out procedure. The study was initiated by the Radiological Society of the Netherlands and the Dutch Surgical Society, in collaboration with the committee which developed the national guidance protocol on preoperative screening for COVID-19, published April 2, 2020. ${ }^{11}$

\section{Study Population}

Consecutive adult patients (18 years or older) who underwent preoperative screening for COVID-19 were included. Patients were eligible for screening if they were scheduled for any type of surgical or interventional procedure under general anesthesia, both elective or emergency, and if they were asymptomatic. Patients scheduled for elective surgery were contacted by telephone 2 to 3 days before surgery and re-checked at admission. Patients undergoing emergency surgery were interviewed at admission. Patients in whom COVID-19 could not be clinically ruled out (ie, incapacitated emergency patients) were not included.

The study consisted of 2 cohorts of consecutive asymptomatic patients. The main cohort were patients who underwent combined screening with CT and RT-PCR. The second and preceding cohort were patients who underwent screening with chest CT only. Most participating centers started preoperative screening with chest CT only. A transition to combined screening occurred in most centers after publication of the national guidance protocol on preoperative screening (April 2nd, 2020), advising centers to screen all preoperative patients using this combined approach. ${ }^{11}$ Participating centers could include patients both retrospectively and prospectively in each cohort. A standard questionnaire was used for evaluation of symptoms in prospectively included patients (see Supplementary Material for symptom questionnaire, http://links.lww.com/SLA/C315). A broad list of symptoms related to COVID-19 were part of the questionnaire. Patients were considered to be asymptomatic if no symptoms suspicious for COVID-19 were present or when symptoms were clearly related to another diagnosis (eg, in cases of fever or abdominal pain in patients with acute appendicitis). See Supplementary Table S1, http://links.lww.com/SLA/C315 on both cohorts' inclusion periods for each participating center.

\section{Data Collection}

Data were extracted on patient's demographics and clinical characteristics, recent exposure history, screening results for chest CT and RT-PCR and operative management. Abbreviated postal codes were collected to explore regional variations in primary outcome.

\section{Chest CT and RT-PCR: Procedure and Analysis}

According to the guidance protocol, preoperative chest CT was performed using an unenhanced low dose protocol. Chest CT scanning could be combined with a contrast-enhanced abdominal 
CT, mostly performed in emergency settings. Chest CT was evaluated by the attending radiologist at each local center and reported using a standard reading protocol. In case of abnormal findings, suspicion for COVID-19 was assessed using the CO-RADS classification. ${ }^{21}$ This classification encodes the level of suspicion for COVID-19 based on chest CT findings (1, very low suspicion; 2, low suspicion, 3, equivocal, 4 , high suspicion, 5 , very high suspicion) and has shown excellent performance for diagnosing COVID-19 in symptomatic patients (average area under the receiver operating curve of $0.91-$ 0.95)..$^{21}$

SARS-CoV-2 RNA detection in nasopharyngeal and/or oropharyngeal swab specimens was performed using RT-PCR assays targeted at the viral envelope, RNA-dependent RNA polymerase and/ or nucleocapsid genes according to nationally endorsed and qualitycontrolled protocols. ${ }^{22}$ For positive RT-PCR specimens cycling threshold values were reported.

A positive screening result for detection of COVID-19 was defined as a CO-RADS score 4 or 5 and/or a positive RT-PCR result. According to the national guideline, in these cases surgery was postponed when possible or, in cases of emergency surgery, additional personal protection equipment and other precautionary measures were taken to prevent nosocomial spread. In patients who were negative at history taking and preoperative screening, use of standard personal protection equipment was considered sufficient. A CORADS 3 (equivocal) test result with a negative RT-PCR result was not regarded as a positive screening result. A decision to postpone surgery in these patients was made at the local clinician's discretion after discussion in a multidisciplinary team meeting. ${ }^{11}$

\section{Study Outcome and Follow-up}

The primary study outcome was the yield of detected COVID19 with chest CT and RT-PCR. Secondary outcomes consisted of the individual yields of chest CT and RT-PCR, the relationship between screening results and differences in community prevalence, and operative management after screening. Two weeks follow-up data were collected for all patients, which consisted of postoperative diagnosis of COVID-19, related complications and intensive care unit admissions.

\section{Relationship to Community Prevalence}

To investigate the relationship between screening results and community prevalence of COVID-19, we stratified patients by their province of residence and screening dates. Publicly available prevalence data were obtained from the Dutch National Institute for Public Health and the Environment. ${ }^{23}$ The number of inhabitants per province in 2020 were obtained from the Central Agency of Statistics in the Netherlands. ${ }^{24}$ We then separately compared the yield for chest CT and RT-PCR in those provinces among patients to the mean daily COVID-19 admissions per 100,000 inhabitants for the same provinces within a 7-day window around each patient screening (from 3 days before screening to 3 days after).

\section{Statistical Analysis}

Screening results were presented as the number and percentage of patients with a positive screening result, with additional percentages and $95 \%$ confidence intervals (CI's). These CI's were calculated using 1000 bootstrapping samples. Anticipating a 2\% yield, we calculated that recruiting 1000 participants would lead to a $95 \% \mathrm{CI}$ around the estimate that would extend from $1.2 \%$ to $3.0 \%$. Analyses were performed with the use of SPSS software, version 26.0.

\section{RESULTS}

\section{Patient Characteristics}

Between March 20, 2020 and April 24, 2020, a total of 2093 asymptomatic patients were included in 14 participating centers: 1224 were screened by a combination of chest CT and RT-PCR and
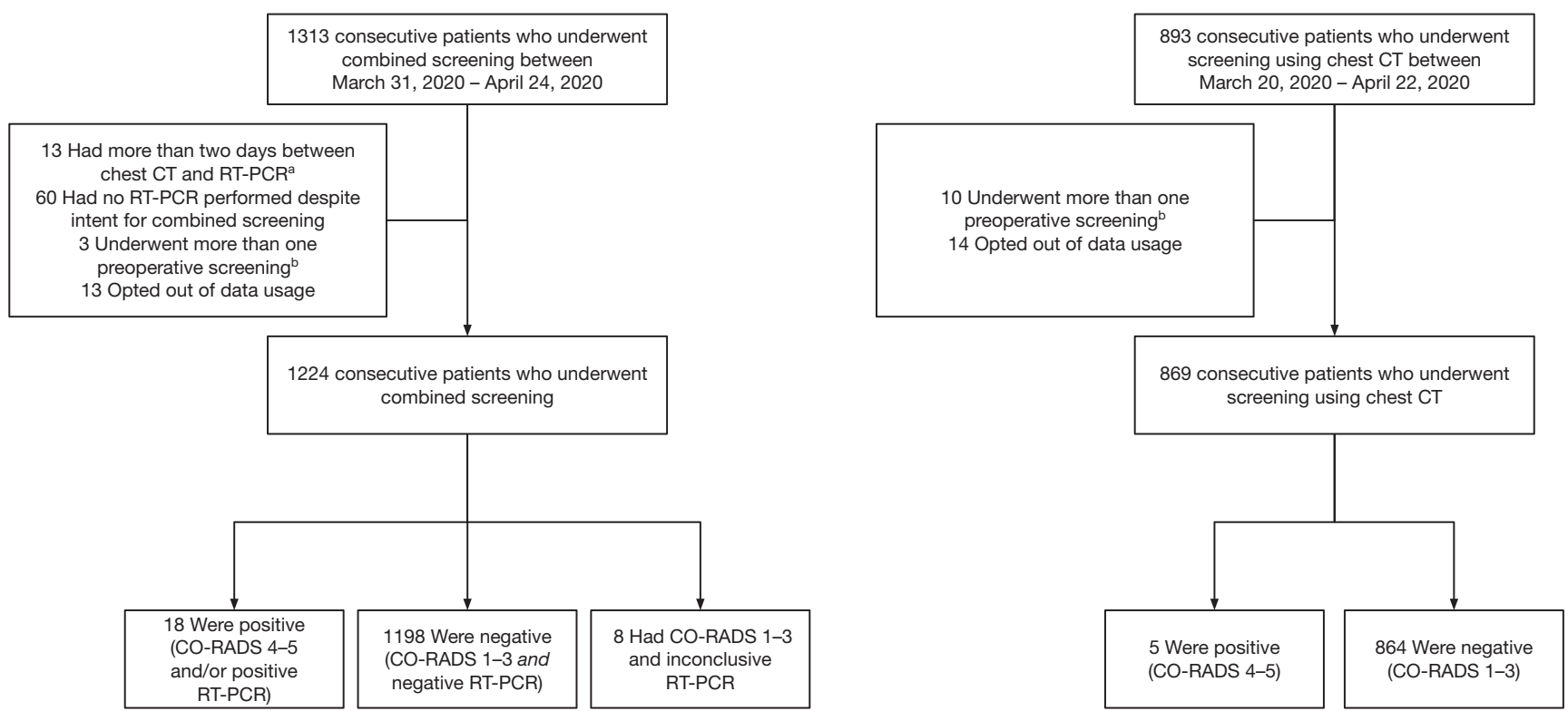

FIGURE 1. Flow-chart for patients undergoing preoperative screening for both cohorts. ${ }^{a}$ These patients all had negative RT-PCR and

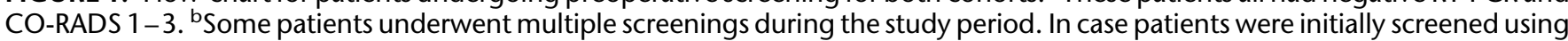
chest $\mathrm{CT}$, and followingly using chest CT and RT-PCR, the combined screening was included. For patients with multiple screenings using 1 strategy, the first screening was included. CT indicates computed tomography; RT-PCR, reverse-transcriptase polymerase chain reaction. 
TABLE 1. Results of Screening With Chest CT and SARS-CoV-2 RT-PCR

\begin{tabular}{|c|c|c|c|}
\hline & \multicolumn{3}{|c|}{ Combined Screening $(n=1224)$} \\
\hline & Chest CT & RT-PCR & Chest CT and RT-PCR \\
\hline $\begin{array}{l}\text { Positive screening result, no./total no.* } \\
\%(95 \% \mathrm{CI})^{\dagger}\end{array}$ & $\begin{array}{c}8 / 1224 \\
0.7(0.2-1.1)\end{array}$ & $\begin{array}{c}14 / 1224 \\
1.1(0.6-1.7)\end{array}$ & $\begin{array}{c}18 / 1224 \\
1.5(0.8-2.2)\end{array}$ \\
\hline
\end{tabular}

869 by chest CT only (Fig. 1). Demographic and clinical characteristics for patients undergoing combined screening are given in Supplementary Table S1, http://links.lww.com/SLA/C315. There were no significant differences in patient characteristics between the 2 cohorts (Supplementary Tables S2 and S3, http://links.lww.com/SLA/C315).

\section{Screening Results}

The results for patients undergoing combined preoperative screening are given in Table 1 . Out of 1224 patients, $18(1.5 \%)$ had positive screening results (Table 2, detailed information in Supplementary Table S4, http://links.lww.com/SLA/C315), all of which were patients scheduled to undergo either elective (14 patients) or semi-urgent surgery (4 patients), but no emergency surgery. Of these patients, $14(1.1 \%)$ tested positive for SARS-CoV-2 using RT-PCR, whereas $8(0.7 \%)$ were suspected for COVID-19 based on chest CT results. Therefore, the incremental yield for chest CT was $0.4 \%$ (4 patients). Concordant positive results were seen in 4 patients with both a positive chest CT result and a positive RT-PCR; 3 of these CT scans were read as CO-RADS 5 (very high suspicion) and 1 as CORADS 4 (high suspicion) (Table 2). When compared against positive RT-PCR results as a reference standard, chest CT had negative findings for 10 of the 14 patients who tested positive for SARSCoV-2 using RT-PCR (71.4\% false negative rate for screening chest CT) (Table 2). Cycling threshold values for positive RT-PCR tests ranged from 20.7 to 37.7 , with a median cycling threshold value of 35.0 (interquartile range 28.3-36.9) (Supplementary Table S4, http:// links.lww.com/SLA/C315). In the second cohort of 869 patients with chest CT only, 5 patients $(0.6 \%$; CI $0.1-1.1)$ had a positive screening result based on chest CT results (all CO-RADS 4).

For combined screening, surgery was postponed because of a positive screening result in 17 patients $(1.4 \%)$. For chest CT only

TABLE 2. Results of Screening With Chest CT and SARS-CoV2 RT-PCR

\begin{tabular}{lccr}
\hline & \multicolumn{3}{c}{ RT-PCR } \\
\cline { 2 - 4 } Chest CT $^{*}$ & Negative/Inconclusive $^{\dagger}$ & Positive & Total \\
\hline Negative & 1090 & 7 & 1097 \\
CORADS 1 & 75 & 2 & 77 \\
CORADS 2 & 41 & 1 & 42 \\
CORADS 3 & & & \\
Positive & 4 & 3 & 5 \\
CORADS 4 & 0 & 14 & 1224 \\
CORADS 5 & 1210 & & \\
Total & &
\end{tabular}

${ }^{*}$ A positive chest CT result was defined as a CORADS 4-5.

tOf 8 patients the screening RT-PCR was inconclusive. Of these patients 7 had CORADS 1 and 1 had CORADS 3 .

CT indicates computed tomography; RT-PCR, reverse-transcriptase polymerase chain reaction. screening, surgery was postponed because of a positive screening result in 2 patients $(0.2 \%)$.

\section{Relationship Between Screening Yield and Community Prevalence}

RT-PCR positive yield was related to the COVID-19 community prevalence: relatively high diagnostic yields up to $\sim 6 \%$ were observed in specific regions and earlier periods where the mean daily COVID-19 hospital admission rate was above 1.5 per 100,000 inhabitants. The yield remained constant at around $\sim 1 \%$ when the hospital admission rate was below 1.5 per 100,000 inhabitants (Fig. 2). There was no relationship between the yield from chest CT screening and COVID-19 community prevalence, which was confirmed in both cohorts (Fig. 2 and Fig. S1, http://links.lww.com/ SLA/C315).

\section{Follow-up Data}

Clinical details for the 18 patients with positive results in combined screening are shown in Supplementary Table S4, http:// links.lww.com/SLA/C315. Two patients with positive screening RTPCR developed COVID-19 symptoms within 2 weeks postoperatively and thus could have been presymptomatic. Two patients with positive screening RT-PCR had been symptomatic within 1 month before screening, but were asymptomatic for at least 2 days before screening, suggesting possible late-stage COVID-19. The remaining 14 patients did not report any COVID-19 related symptoms before, during or after screening. These included the 4 patients who had positive chest CT (all CO-RADS 4) with negative RT-PCR results (Supplementary Table S4, http://links.lww.com/SLA/C315). Of the 5 patients who had positive screening results in the chest CT only cohort, 3 of these 5 underwent RT-PCR testing with negative results at 1,1 and 11 days after screening. Two of these patients were tested because of the CO-RADS 4 score at screening, while a third patient was tested because of a newly scheduled surgical procedure. None of the 5 chest $\mathrm{CT}$ positive patients in the chest CT only cohort developed COVID-19 symptoms within 2 weeks after chest CT screening.

Of 1206 patients who underwent combined screening with negative screening results, 1169 patients underwent surgery, of which none developed symptomatic COVID-19 within 2 weeks postoperatively. Of 864 patients who underwent screening using chest CT only with negative screening results, 829 patients underwent surgery of which 3 developed symptomatic COVID-19 postoperatively, diagnosed by positive RT-PCR at 1, 5, and 7 days after surgery. None of these 3 patients were initially screened using RTPCR. None of them required admission to the intensive care unit postoperatively because of COVID- 19 .

\section{DISCUSSION}

This is the first multicenter study to determine the yield of screening for COVID-19 using chest CT and RT-PCR in asymptomatic patients before elective or emergency surgery. Combined 


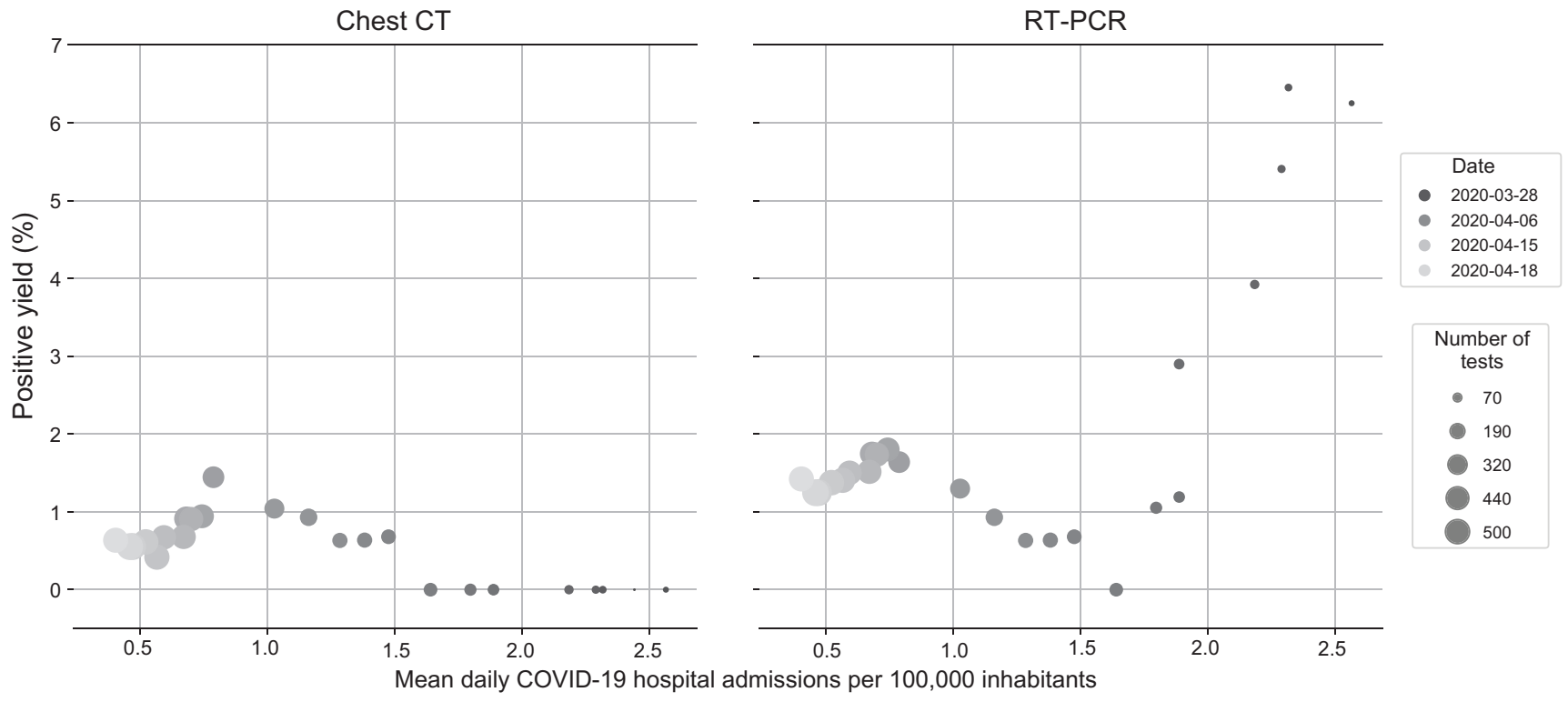

FIGURE 2. Association of positive yield for chest CT and SARS-CoV-2 RT-PCR of asymptomatic patients with regional and temporal variations in community prevalence, measured by the mean daily COVID-19 related hospital admissions per 100.000 inhabitants. Left panel: no discernable pattern of association between positive chest CT results and regional and temporal variations of COVID-19 prevalence. Right panel: positive yield by RT-PCR markedly increases above mean of 1.5 daily COVID-19 related hospital admissions per 100.000 inhabitants. CT indicates computed tomography; RT-PCR, reverse-transcriptase polymerase chain reaction.

preoperative screening demonstrated a yield of $1.5 \%$, of which RTPCR confirmed SARS-CoV-2 infection in $1.1 \%$ of patients. Chest CT showed an incremental yield of $0.4 \%$, although these could be falsepositive results as none of these patients developed COVID-19 symptoms and no relationship with community prevalence was seen for chest CT results. No postoperative symptomatic COVID-19 infections were seen in patients who had negative RT-PCR screening results. In contrast, 3 patients who underwent screening using only chest CT with negative results developed postoperative symptomatic COVID-19, suggesting that these infections might have been missed by CT.

Two other studies have investigated the use of RT-PCR as a screening method in asymptomatic patients, but none in patients undergoing surgery or other interventions under general anesthesia. ${ }^{25,26}$ Data from both previous studies confirm the association between yield of RT-PCR in asymptomatic patients and number of COVID-19 related hospital admissions. A study from New York City found SARS-CoV-2 infection in $13.7 \%$ of 210 asymptomatic women admitted for delivery when the average of daily COVID-19 related hospital admissions was around 16.0 per 100.000 inhabitants. ${ }^{25,27} \mathrm{In}$ Iceland, screening of a random population sample using RT-PCR found that 13 of $2283(0.6 \%)$ individuals were positive for SARSCoV-2. However, 6 of these 13 individuals reported COVID-19 related symptoms. ${ }^{26}$ During this period, the average daily COVID-19 related hospital admissions in Iceland was around 0.78 per 100.000 inhabitants. ${ }^{28}$ These data confirm the association between the RT-PCR yield in asymptomatic patients and hospital admissions, as both screening yield and the hospital admission rate were around 20 times higher in New York City as compared to the Iceland population. These, and our findings also suggest that the number of patients with asymptomatic COVID-19 is higher than previously reported. ${ }^{29,30}$

Social distancing measures (limited lock down) were implemented by the Dutch government on March 12, 2020. Peak prevalence for COVID-19 in the Netherlands occurred during the second half of March after which prevalence decreased. Although preoperative screening was initiated in several hospitals from March 23 onwards, combined screening with chest CT and RT-PCR was implemented starting on March 31, 2020. As community prevalence decreased in the Netherlands from $\sim 3$ to $\sim 1.5$ COVID-19 related hospital admissions per 100,000 inhabitants, the RT-PCR yield in our study patients decreased markedly from potentially as high as $\sim 6 \%$ to $\sim 1 \%$. The screening yield remained at $\sim 1 \%$ as admission rate decreased to $\sim 0.5$ per 100,000 inhabitants. The absent relationship between positive chest CT findings and COVID-19 community prevalence further questions the sensitivity of these positive CT findings as related to asymptomatic COVID-19.

Preoperative screening with RT-PCR found infection with SARS-CoV-2 in only $1.1 \%$ of patients. Although this yield may seem low, even a small number of undetected cases could have substantial consequences. Data on the impact of COVID-19 on patients undergoing surgery is scarce. Lei et al (2020) described postoperative outcomes in a group of 34 patients undergoing surgery, all of whom developed symptomatic COVID-19 within 4 days postoperatively and were; therefore, considered to have been in their incubation period before surgery. ${ }^{3}$ Fifteen patients required admission to the intensive care unit $(44.1 \%)$, whereas 7 patients died $(20.5 \%)$ postoperatively, both considerably higher than previously reported for hospitalized nonsurgical COVID-19 patients. These findings indicate the importance of preoperative screening to prevent adverse postoperative outcomes.

Additionally, asymptomatic patients with SARS-CoV-2 could be shedders of the virus, especially during aerosol generating procedures, thereby placing other patients and hospital workers at risk, especially those performing endotracheal intubation. One study evaluated transmission of SARS-CoV-2 within a skilled nursing facility, by RT-PCR testing on 2 occasions as part of a facility-wide point-prevalence survey. ${ }^{27}$ Forty-eight out of seventy-six $(63.0 \%)$ 
residents tested positive for SARS-CoV-2. Of those 48 residents, 27 $(56 \%)$ were asymptomatic at time of testing. SARS-CoV-2 could rapidly spread through the skilled nursing facility to other residents and staff, probably due to unrecognized asymptomatic and presymptomatic infections. These results indicate symptom-based screening to be insufficient to prevent rapid transmission in skilled nursing facilities. In our study the median RT-PCR cycle threshold value of the positive PCRs was relatively high, suggesting a low viral load, which may affect the risk for transmission. ${ }^{31}$ During aerosol generating procedures such as intubation; however, the risk of transmission is probably increased. ${ }^{32}$ By avoiding introduction of COVID-19 positive patients into the hospital, preoperative screening benefits surgical care in preventing nosocomial spread and reducing the use of scarce personal protective equipment.

Our study has some limitations. First, patients undergoing preoperative screening using only chest CT were mostly included retrospectively. Preoperative symptoms were not inquired using a standardized procedure in these patients. Some patients with mild unrecognized complaints could; therefore, have been unknowingly included. Second, the study was conducted during the quarantine period in the Netherlands, which effectively commenced on March 15th, 2020. Consequently, we experienced a decreasing prevalence during the inclusion period, which may have led to a decreasing yield. This could have affected the accuracy of our positive yield and relationship to community prevalence. Third, a relatively low number of RT-PCR screenings were performed early in the study, when new COVID-19 hospital admissions in the Netherlands were at their highest level. ${ }^{23}$ As such, the high positivity rates in the first period could be inflated due to small sample bias. Fourth, no analysis for risk factors could be performed due to the limited number of patients with positive results. Last, we used the daily hospital admission rate as a marker for COVID-19 community prevalence in the population. Although a relationship was found, the exact cut-off value of 1.5 daily admissions per 100,000 inhabitants above which the screening yield increases could vary due to differences between national health care systems, such as the availability of pre-hospital care (eg, general physicians). Moreover, our study population consisted of surgical patients and thus was not fully representative for the general population.

Preoperative screening with RT-PCR found infection with SARS-CoV-2 in at least 1 in every 100 asymptomatic patients, increasing in conjunction with community prevalence. Given the limited added value, the use of chest $\mathrm{CT}$ in preoperative screening is not recommended based on our results. Preoperative screening in asymptomatic COVID-19 patients undergoing surgery should be performed with RT-PCR. The initiation of preoperative screening can be directed by local community prevalence of COVID-19.

\section{REFERENCES}

1. Cobianchi L, Pugliese L, Mis F, et al. To a new normal: surgery and COVID-19 during the transition phase. Ann Surg. 2020. Epub ahead of print.

2. Liang ZC, Chong MSY, Liu GKP, et al. COVID-19 and elective surgery. Ann Surg. 2020. Epub ahead of print.

3. Besnier E, Tuech J-J, Schwarz L. We asked the experts: Covid-19 outbreak: is there still a place for scheduled surgery? "Reflection from pathophysiological data. World J Surg. 2020;44:1. doi:10.1007/s00268-020-05501-6.

4. Lei S, Jiang F, Su W, et al. Clinical characteristics and outcomes of patients undergoing surgeries during the incubation period of COVID-19 infection. EClinicalMedicine. 2020;21. Epub ahead of print.

5. Aminian A, Safari S, Razeghian-Jahromi A, et al. COVID-19 outbreak and surgical practice: unexpected fatality in perioperative period. Ann Surg. 2020;272:e27-e29.

6. Kluytmans-van den Bergh M, Buiting A, Pas S, et al. SARS-CoV-2 infection in 86 healthcare workers in two Dutch hospitals in March 2020. medRxiv. 2020;20041913. doi:10.1101/2020.03.23.20041913.
7. Chan JFW, Yuan S, Kok KH, et al. A familial cluster of pneumonia associated with the 2019 novel coronavirus indicating person-to-person transmission: a study of a family cluster. Lancet. 2020;395:514-523.

8. Hu Z, Song $\mathrm{C}$, Xu C, et al. Clinical characteristics of 24 asymptomatic infections with COVID-19 screened among close contacts in Nanjing, China. Sci China Life Sci. 2020;63:706-711.

9. Hains DS, Schwaderer AL, Carroll AE, et al. Asymptomatic seroconversion of immunoglobulins to SARS-CoV-2 in a pediatric dialysis unit. JAMA. 2020;323:2424-2425.

10. Guan W, Ni Z, Hu Y, et al. Clinical characteristics of coronavirus disease 2019 in China. N Engl J Med. 2020;382:1708-1720.

11. LEIDRAAD Pre-Operatieve Diagnostische Work Up. Available at: https:// heelkunde.nl/sites/heelkunde.nl/files/Practice\%20Guideline\%20Preoperative\%20work\%20up\%20on\%20possible\%20COVID-19\%20infection\%20in\%20asymptomatic\%20patients.pdf. Accessed April 29, 2020.

12. Oba A, Stoop TF, Lohr M, et al. Global survey on pancreatic surgery during the COVID-19 pandemic. Ann Surg. 2020. Epub ahead of print.

13. Bhimraj A, Morgan RL, Shumaker AH, et al. Infectious Diseases Society of America guidelines on the treatment and management of patients with COVID-19. Clin Infect Dis. 2020. Epub ahead of print.

14. Woelfel R, Corman VM, Guggemos W, et al. Virological assessment of hospitalized cases of coronavirus disease 2019. medRxiv. 2020;20030502. doi:10.1101/2020.03.05.20030502.

15. Ai T, Yang Z, Xia L. Correlation of chest CT and RT-PCR testing in coronavirus disease. Radiology. 2020;2019:1-8.

16. Fang Y, Zhang H, Xie J, et al. Sensitivity of chest CT for COVID-19: comparison to RT-PCR. Radiology. 2020;200432. doi:10.1148/ radiol.2020200432.

17. Liu R, Han H, Liu F, et al. Positive rate of RT-PCR detection of SARS-CoV-2 infection in 4880 cases from one hospital in Wuhan, China, from Jan to Feb 2020. Clin Chim Acta. 2020;505:172-175.

18. Rubin GD, Haramati LB, Kanne JP, et al. The role of chest imaging in patient management during the COVID-19 pandemic: a multinational consensus statement from the Fleischner society. Radiology. 2020;S0012-3692. doi:10.1148/radiol.2020201365.

19. Inui S, Fujikawa A, Jitsu M, et al. Chest CT findings in cases from the cruise ship "diamond princess" with coronavirus disease 2019 (COVID-19). Radiol Cardiothorac Imaging. 2020;2:e200110.

20. Wang Y, Dong C, Hu Y, et al. Temporal changes of CT findings in 90 patients with COVID-19 pneumonia: a longitudinal study. Radiology. 2020. Epub ahead of print.

21. Prokop M, van Everdingen W, van Rees Vellinga T, et al. CO-RADS - a categorical CT assessment scheme for patients with suspected COVID-19: definition and evaluation. Radiology. 2020. Epub ahead of print.

22. Corman VM, Landt O, Kaiser M, et al. Detection of 2019 novel coronavirus (2019-nCoV) by real-time RT-PCR. Eurosurveillance. 2020;25:2000045. doi:10.2807/1560-7917.ES.2020.25.3.2000045.

23. Rijksinstituut voor Volksgezondheid en Milieu | RIVM. Available at: https:// www.rivm.nl/. Accessed April 29, 2020.

24. CBS StatLine. Available at: https://opendata.cbs.nl/\#/CBS/nl/. Accessed April 29, 2020.

25. Sutton D, Fuchs K, D'Alton M, et al. Universal screening for SARS-CoV-2 in women admitted for delivery. N Engl J Med. 2020;382:2163-2164.

26. Gudbjartsson DF, Helgason A, Jonsson H, et al. Spread of SARS-CoV-2 in the Icelandic population. $N$ Engl J Med. 2020;382:2302-2315.

27. Welcome to NYC.gov | City of New York. Available at: https://www1.nyc.gov/. Accessed April 29, 2020.

28. COVID-19 in Iceland. Available at: https://covid.hi.is/. Accessed April 29, 2020.

29. Arons MM, Hatfield KM, Reddy SC, et al. Presymptomatic SARS-CoV-2 infections and transmission in a skilled nursing facility. $N$ Engl $\mathrm{J} \mathrm{Med}$. 2020;382:2081-2090.

30. Mizumoto K, Kagaya K, Zarebski A, et al. Estimating the asymptomatic proportion of coronavirus disease 2019 (COVID-19) cases on board the Diamond Princess cruise ship, Yokohama, Japan, 2020. Eurosurveillance. 2020;25:2000180.

31. Chu HY, Englund JA, Starita LM, et al. Early detection of Covid-19 through a citywide pandemic surveillance platform. $N$ Engl $J$ Med. 2020;323:NEJMc2008646. doi:10.1056/NEJMc2008646.

32. Weissman DN, de Perio MA, Radonovich LJ. COVID-19 and risks posed to personnel during endotracheal intubation. JAMA. 2020. Epub ahead of print. 